\title{
Parallele Universen oder Verschmelzung der Horizonte?
}

Der Beitrag argumentiert, dass sich Internationale Politische Theorie vorrangig über ihren empirischen Gegenstand formiert und auch definieren sollte: der Verschränkung der Erkenntnisinteressen zwischen den Teildisziplinen als Resultat der Transformation grenzüberschreitender Politik in eine politische Herrschaftsordnung. Stellt man diese Transformation in den Mittelpunkt, folgt daraus, dass Internationale Politische Theorie weder der einen noch der anderen Teildisziplin oder einem spezifischen methodisch-theoretischen Zugang zugeschlagen werden sollte. Vielmehr fordert sie gerade die Pluralität und Verschränkung der Zugänge, um den Herausforderungen, die sich aus der Praxis grenzüberschreitender Politik ergeben, empirisch, begrifflich und normativ begegnen zu können.

\section{Einleitung ${ }^{1}$}

Internationale Politische Theorie (IPT) ist zumindest im deutschsprachigen Raum ein offenes und - wie auch die hier versammelten Symposiumsbeiträge zeigen - umstrittenes Forschungsfeld. Strittig ist sowohl, innerhalb welcher Teildisziplin dieses Forschungsfeld beheimatet sein sollte, Politischer Theorie oder Internationaler Beziehungen als auch, welche theoretischen und methodischen Zugänge das Feld definieren sollten. Aus ihren jeweiligen Teildisziplinen heraus beschreiben Antje Wiener (2010, in diesem Heft) und Peter Niesen (2010, in diesem Heft) eine Internationale Politische Theorie eher als Erweiterung des Forschungsprogramms der jeweiligen Teildisziplin. Hauke Brunkhorst (2010, in diesem Heft) und mehr noch Oliver Kessler (2010, in diesem Heft) plädieren dagegen für eine Neubestimmung des Felds jenseits der beiden Teildisziplinen, während Rainer Forst (2010, in diesem Heft) IPT als Feld fruchtbaren Austauschs zwischen Politischer Theorie und Internationalen Beziehungen sieht. ${ }^{2}$ Ähnlich uneins sind sich die Beiträge über die theoretisch-methodische Verortung der IPT, ob sie als Ausdruck normativer Theorie im engeren (Niesen 2010) und weiteren Sinne (Wiener 2010) zu verstehen ist oder nach einer - neuen - gesellschaftstheoretischen Fundierung verlangt (Kessler 2010; Brunkhorst 2010).

Im Gegensatz zu den anderen Beiträgen (vgl. aber Forst 2010) will ich im Folgenden argumentieren, dass sich Internationale Politische Theorie vorrangig über ihren empirischen Gegenstand formiert und auch definieren sollte: der Praxis grenzüber-

1 Für vielfältige Anregungen und Kommentare danke ich den Mitgliedern der DissidenzForschungsgruppe, Christopher Daase, Christian Volk, Sebastian Schindler und Thorsten Thiel sowie den Gutachterinnen und Gutachtern.

2 Obgleich Forst (2010) von einer Zusammenarbeit zwischen Internationaler Politischer Theorie und IB spricht und damit letztlich offen lässt, ob die IB nun Teil der IPT oder nur deren Kooperationspartner ist. 
schreitender Politik (ähnlich auch Niesen 2010). Stellt man diese Praxis in den Mittelpunkt, folgt daraus, dass Internationale Politische Theorie weder der einen noch der anderen Teildisziplin oder einem spezifischen methodisch-theoretischen Zugang zugeschlagen werden sollte. Vielmehr fordert sie gerade die Pluralität und Verschränkung der Zugänge, um den Herausforderungen, die sich aus der Praxis grenzüberschreitender Politik ergeben, empirisch, begrifflich und normativ begegnen zu können.

Konkret werde ich daher argumentieren, dass sich das Feld einer Internationalen Politischen Theorie aus der Verschränkung der Erkenntnisinteressen zwischen den Teildisziplinen speist, die ihrerseits - zumindest auch - Resultat der Transformation grenzüberschreitender Politik in eine politische Herrschaftsordnung ist (Abschnitt 2). Angesichts dieser Entwicklung wird die Sphäre grenzüberschreitender Politik innerhalb der Politischen Theorie zunehmend notwendiger Anwendungsbereich, um die ihr eigenen Fragen nach der Wirkmächtigkeit politischer Ideen, der Legitimationsfähigkeit politischer Ordnungen und ihrer Institutionen zu thematisieren. Umgekehrt werden genau diese klassischen Fragen der Politischen Theorie in den Internationalen Beziehungen immer zentraler, um den eigenen Gegenstand $\mathrm{zu}$ bearbeiten (Abschnitt 3). Hieraus folgt jedoch (noch) keine integrierte Forschungsgemeinschaft oder gar eine Verschmelzung der Horizonte (Gadamer 1990); es bleibt, zumindest in Deutschland, bislang ein amorphes Forschungsfeld. ${ }^{3}$ So wünschenswert es ist, die Verschränkung der Erkenntnisinteressen weiter voranzutreiben, so wichtig ist es zugleich, den gegenwärtigen Diskurs zwischen den Teildisziplinen - oder genauer zwischen den jeweiligen Teil-communities ${ }^{4}$ in den Teildisziplinen über eine Internationale Politische Theorie nicht durch vorzeitige, einseitige Grenzziehungen und Vereinnahmungen zu verengen, ${ }^{5}$ denn genau in diesem produktiven Streit, in der Konfrontation unterschiedlicher begrifflicher, analytischer und normativer Zugänge wird m.E. das Potenzial für neue Fragen und Erkenntnisse erschlossen. Internationale Politische Theorie verstehe ich darum - im Gegensatz etwa zu Kessler (2010) - ganz bewusst als Schnittstelle zwischen Politischer Theorie und Internationalen Beziehungen. ${ }^{6}$ Gefordert ist die Verschmelzung der Erkenntnishorizonte, nicht aber der methodischen oder theoretischen Zugänge (Abschnitt 4).

3 In anderen nationalen Kontexten, wie etwa Großbritannien, ist dagegen das Feld der Internationalen Politischen Theorie recht klar definiert. Vgl. dazu den Beitrag von Niesen (2010).

4 Diese Einschränkung erscheint mir wesentlich, denn natürlich ist weder für die Politische Theorie noch für die Internationalen Beziehungen das Feld Internationaler Politischer Theorie das einzige - oder auch nur primäre - Forschungsfeld.

5 Solche Grenzziehungen lassen sich etwa für die ECPR standing group zu International Political Theory beobachten, die das Feld von vornherein auf Internationale Politische Philosophie und Internationale Ethik beschränken. Vgl. dazu die Kritik bei Forst (2010).

6 Kessler argumentiert gegen eine »Schnittstelle«, weil diese zu einer Verengung des Feldes führen würde, insofern die unterschiedlichen Logiken der beiden Teildisziplinen, Internationaler Beziehungen (empirisch-sozialwissenschaftlich) und Politischer Theorie (begrifflich) notwendig dazu führen müssten, dass nur eine Teilmenge von in beiden Teildisziplinen breit konsentierten Konzepten und Denkern thematisiert würde (Kessler 2010). Warum diese Verengung allerdings notwendig folgt, bleibt unklar. 


\section{Grenzüberschreitende Politik als Herrschaftsordnung}

»Das erste, womit das Verstehen beginnt, ist, dass uns etwas anspricht« (Gadamer 1993: 64).

Das Moment des »uns Ansprechens« scheint mir eine sinnvolle Beschreibung zu sein, um die Entwicklung hin zu einer Internationalen Politischen Theorie zu verstehen. Gadamer versteht unter dem Moment des Ansprechens die Irritation durch die Begegnung mit etwas Befremdlichen, Desorientierendem, das unsere bestehenden Urteile und Schlüsse herausfordert (Gadamer 1993: 185). Ganz ähnlich lässt sich die Internationale Politische Theorie als Ergebnis einer Irritation verstehen, die innerhalb beider Teildisziplinen hinsichtlich ihres Forschungsgegenstands wahrgenommen und bestenfalls wechselseitig, gegenwärtig noch zu häufig parallel, problematisiert wird.

Diese Irritation kommt dadurch zustande, dass sich die Gegenstände beider Teildisziplinen zumindest teilweise aus ihren angestammten Kontexten herauszulösen scheinen. Für die Politische Theorie, unabhängig davon, ob sie sich als normative oder empirische Politische Theorie, als Demokratiewissenschaft oder als politische Philosophie versteht (vgl. Niesen 2007; Brodocz/Schaal 2002), ist dies genauso der staatliche Kontext wie für die Internationalen Beziehungen. Fragt nach Göhler et al. (2009: 373) die Politische Theorie »von jeher nach der Ordnung des Zusammenlebens der Menschen in einem Gemeinwesen«, kann für die Forschungspraxis wohl unumstritten gelten, dass dieses Gemeinwesen üblicherweise mit dem Staat gleichgesetzt wurde. Es ging um die phänomenologische Erfassung der Ordnungsmuster, ihrer institutionellen Verfasstheit und die Frage der legitimen normativen Ordnung im Staat (Deitelhoff/Steffek 2009). ${ }^{7}$

Im Gegensatz zur Politischen Theorie stand für die Internationalen Beziehungen lange nicht die Ordnung, geschweige denn die legitime Ordnung in einem Gemeinwesen, im Kern des Erkenntnisinteresses, sondern die Frage, wie die Koexistenz von souveränen Staaten so zu gestalten wäre, dass der Frieden zwischen ihnen gesichert würde (Rittberger 1990: 8). ${ }^{8}$ Dennoch bildete auch hier der staatliche Kontext den Bezugspunkt. Zwar fokussieren die Internationalen Beziehungen auf die Politik jenseits des Staates, sie setzten diesen aber zugleich lange als unproblematischen Bezugspunkt voraus. Internationale Beziehungen waren in erster Linie zwischenstaatliche Beziehungen. Und schon der Ausdruck »Beziehungen« verdeutlicht, dass man auch kaum von normativer Ordnung auszugehen können glaubte (vgl. aber die Englische Schule: Bull 1977; Daase 2003 im Überblick). Internationale Ordnung, wenn denn der Begriff bemüht wurde, beschrieb eine spezifische Machtordnung, die stabil zu halten war, um den Ausbruch kriegerischer Gewalt zu vermeiden. Ziel internationaler Ordnung, d.h. der Regeln, Normen und Institutionen war die friedliche Koexis-

7 Obschon es, wie Forst (2010) betont, durchaus immer Arbeiten zu Politik jenseits des Staates gab.

8 Allerdings ist diese Unterscheidung heute kaum noch zu halten. Der klassische Friedensfokus der Internationalen Beziehungen wurde mit der Herausbildung des governance-Paradigmas zunächst von einem Problemlösungs- bzw. Steuerungsfokus und mittlerweile von einem Fokus auf demokratische Legitimität überlagert (vgl. dazu Deitelhoff/Wolf 2009: 458-459). 
tenz der Staaten (Ruggie 1983). Daraus ergaben sich aber für die Internationalen Beziehungen weder eine Vergesellschaftung oder Vergemeinschaftung noch eine Herrschaftsordnung auf internationaler Ebene (vgl. hierzu ausführlicher Kessler 2010 und Brunkhorst 2010). Herrschaft fand innerhalb der Staaten statt, während die internationale Ebene diese durch Koordinationsnormen abzusichern hatte (Steffek 2009: 167). Genau an diesem letzten Punkt, dem Fehlen von Herrschaft auf der internationalen Ebene, entbindet sich die Irritation, denn immer häufiger wird die Ebene internationaler, jetzt eher globaler Politik ${ }^{9}$ als Sphäre einer politischen Herrschaftsordnung begriffen, d.h. im Gegensatz zu einer Machtordnung, die sie immer war, als ein dichtes Mehrebenensystem institutionalisierter Über- und Unterordnungsverhältnisse (vgl. Hurrell 2007).

Mit dieser Form von Herrschaftsordnung verbindet sich allerdings keine einheitliche Unterworfenheit unter Regeln, d.h. kein Zentrum der Herrschaft, das einen klaren Adressaten normativer Kritik oder von Widerstand benennen würde. Das Idealbild eines hierarchisch strukturierten Herrschaftsverbands im Weberschen Sinne (Weber 1972) hat sich jenseits des Nationalstaats nicht herausgebildet. Stattdessen lassen sich Merkmale ausmachen, die eher einer heterarchischen Herrschaftsordnung entsprechen (vgl. zu Heterarchie in den IB Neyer 2002; 2004). Heterarchische Ordnungen zeichnen sich nicht durch klassische Befehls-Gehorsams-Korrelationen aus (Arendt 1970), sondern beschreiben die dezentralisierten Verfahren in Systemen, in denen Herrschaft sich in konkurrierenden, zumindest aber überlappenden Entscheidungsund Machtzentren manifestiert. Macht tritt darin nicht als direkte Beherrschung durch mächtige Akteure zutage, sondern manifestiert sich im asymmetrischen Zugang zu und Einfluss auf soziale und politische Systembildungs- und Steuerungsprozesse(n) (Deutsch 1969; Luhmann 2003). Diese Charakteristika lassen sich für die veränderte Praxis globaler Politik eindrücklich beobachten.

Mit der Zunahme grenzüberschreitender Interaktionen im Zuge von Globalisierungs- oder Denationalisierungsprozessen (Zangl/Zürn 2003; Beck 2007), wie wir sie spätestens seit den 1980er Jahren beobachten können, hat sich die Gestalt grenzüberschreitender Politik erheblich gewandelt. Der steigende Bedarf an grenzüberschreitenden Regulierungen hat zu einer immensen Ausweitung und Vertiefung internationaler Normen und Institutionen geführt, die immer tiefer in die nationale Herrschaftsordnungen eingreifen und längst auch von nichtstaatlichen Regelungsadressaten Anpassungsleistungen und Folgebereitschaft erfordern (Kahler 1995), ohne dass zugleich mit diesen Verregelungs- und Verrechtlichungsschüben die Prozeduren einer legitimen Meinungs- und Willensbildung hätten mithalten können (Brock 2007: 47-48). Internationale Institutionen nehmen einerseits vermehrt supranationale Züge an, während andererseits private Akteure in die Regelsetzung und -umsetzung drängen oder Politiken in neue, eher informelle Institutionen abwandern (Held/McGrew 2002: 6). Nimmt man dazu noch die weiter aufklaffende Wohlstandsschere zwischen Globalem Norden und Globalem Süden (Kapstein 2000; Higgott 2000), die die be-

9 Ich verwende »global«, um deutlich zu machen, dass sich grenzüberschreitende Politik mittlerweile nicht mehr auf zwischenstaatliche Arrangements begrenzen lässt. 
stehenden Ungleichheiten zwischen den Staaten befeuert, wird deutlich, dass das Bild einer internationalen Politik als System horizontaler Koordination, das auf Staatenkonsens beruht, kaum noch zu halten ist (Buchanan/Keohane 2006: 407; Hurrell 2007), sondern dass sich globale Politik zunehmend als eine - wenngleich fragmentierte, uneinheitliche heterarchische - Herrschaftsordnung zu strukturieren beginnt, die die Funktionsfähigkeit nationaler Herrschaftsordnungen nicht nur unterläuft, sondern partiell auch zu überschreiben beginnt und damit die Grundfragen politischer Ordnungsbildung und -reproduktion, ihrer Legitimation und Kritik in die Sphäre des Internationalen überträgt (vgl. Müller 2008; als Ausdruck weltgesellschaftlicher Prozesse bei Brunkhorst 2010).

Die Irritation ob des Herrschaftscharakters globaler Politik lässt sich vielerorts beobachten. Bereits im Call for Papers für die Frankfurter Tagung (Deitelhoff et al. 2010) ist die Sprache vom »politischen Bereich jenseits der Einzelstaaten, der unser aller Leben heute wesentlich mitbestimmt « (Stefan Gosepath), oder von »grenzüberschreitenden Governance- und Government-Arrangements« (Peter Niesen; meine Hervorhebung). Auch in der Forschungs- und Publikationspraxis hinterlässt sie längst Spuren. Plädierte etwa Harald Müller noch 2004 in einem Forum zu den Konsequenzen von 9/11 für die Internationalen Beziehungen in dieser Zeitschrift für die dringende Reintegration der Ethik in die Internationalen Beziehungen, die Wiederbeschäftigung mit normativen Fragen von Gerechtigkeit, kultureller Diversität und der Frage von Anerkennungsprozessen (Müller 2004: 130-131), so ist inzwischen die Fülle von Publikationen zu Fragen globaler Gerechtigkeit, der demokratischen Legitimität globalen Regierens oder den Grundlagen globaler Vergesellschaftung auch innerhalb der Internationalen Beziehungen kaum noch zu überblicken. ${ }^{10}$ Allerdings charakterisiert sich das Gros dieser Arbeiten eher durch das Plädoyer für die gezielte Errichtung legitimer Herrschaftsstrukturen auf globaler Ebene, als dass die bereits bestehende Herrschaft in ihren Grundlagen und Konsequenzen analysiert würde. ${ }^{11}$ Explizite Analysen des Herrschaftscharakters globaler Politik bleiben bislang in der Domäne poststrukturalistischer und postmarxistischer Perspektiven auf Global Governance (etwa Hardt/Negri 2001; Gill 2003), die hauptsächlich generelle Herrschafts- und Ideologiekritik betreiben. Dennoch lässt sich die Prominenz, die diese

10 Vgl. unter vielen anderen etwa die Sammelbände von Niesen/Herborth (2007); Brunkhorst (2009); Hurrelmann et al. (2007); Albert/Stichweh (2007); Kohler-Koch/Rittberger (2007). Natürlich gab es schon zuvor Arbeiten im Bereich Internationale Politische Theorie, insbesondere zu Fragen der demokratischen Legitimität des Regierens jenseits des Staates (vgl. etwa Zürn 1998; Wolf 2000). In Deutschland ist dabei auffällig, dass die Arbeiten zumeist empirisch-analytische Auseinandersetzungen mit normativen Konzepten darstellen (vgl. Deitelhoff/Wolf 2009), so etwa in der ZIB-Debatte zu Beginn der 1990er Jahre über den Import der Gesellschaftstheorie von Jürgen Habermas in die Internationalen Beziehungen (Müller 1994; Schmalz-Bruns 1995; vgl. aber etwa auch Hasenclever 2002 zu Macht und Moral in den internationalen Beziehungen; Mayer 2006 zu Gerechtigkeit und Kooperation), während normative Arbeiten oder Internationale Ethik eher im angelsächsischen Raum dominieren, hier häufig auch zeitlich sehr viel früher präsent waren (vgl. etwa Frost 1986, Linklater 1998; Brown 1992; Beitz 1979). Vgl. dazu auch den Beitrag von Peter Niesen (Niesen 2010: 269-271). Kessler 2010 zieht dagegen daraus den Beleg, dass die IB sich generell über die empirische Forschung definiert.

11 Ich danke den Gutachterinnen und Gutachtern für diesen Hinweis. 
Ansätze inzwischen erhalten, kaum verstehen, ohne zu unterstellen, dass globale Politik zumindest auch als Herrschaftsordnung begriffen wird.

Gleichwohl lässt sich natürlich fragen, ob wir hier tatsächlich etwas Neues beobachten oder ob wir es nicht nur als neu wahrnehmen. Die Politikwissenschaft ist bekanntlich voll vom ewig gleichen Neuen, erinnert sei nur an die Debatte um die Neuen Kriege (Münkler 2002) oder den Neuen Regionalismus (Hettne/Söderbaum 2008). In gewissem Sinne ist internationale Politik immer schon zugleich Herrschaftsordnung gewesen, insofern die Anerkennung als Mitglieder dieser Ordnung immer mit Verhaltensanforderungen verknüpft war und es immer schon Subordinationsverhältnisse zwischen Machtzentren und Peripherien gab. Auch die Frage, ob das gegenwärtige Ausmaß an Globalisierung tatsächlich ein historisches Hoch darstellt, ist nach wie vor umstritten (Hirst/Thompson 1996; vgl. auch Osterhammel/Petersson 2004).

Warum sich gleichwohl zumindest die Wahrnehmung globaler Politik zu verändern scheint, könnte mehrere Gründe haben. Ein Faktor mag die mangelnde Durchgriffstiefe internationaler Normen und Institutionen sein. Unter Bedingungen friedlicher Koexistenz war das internationale System eher durch Regeln geprägt, die auftretende Koordinationsprobleme zwischen Staaten regulieren sollten, aber die Autonomie der Staaten weitgehend unangetastet ließen (Zangl/Zürn 2003; Ruggie 1983). Gleichwohl ist zu konzedieren, dass etwa die Praxis der Kolonialpolitik oder der Sicherheitspolitik der europäischen Mächte im 18. Jahrhundert (Durchhardt 1997: 4), wenngleich in variierendem Maße, kaum zu unterschätzende Effekte auf die Lebenschancen von Staaten generell zeitigte. Gravierender mag indes sein, dass diese Form der Herrschaft zentral an ihren Rändern auftrat (in Fragen, wer mit welchen Rechten überhaupt Mitglied ist) und dass sie kaum an allen Orten in gleicher Weise auftrat (Europäische Mächte und außereuropäische und nicht-christliche Staaten) noch wahrgenommen wurde. Bedeutsamer ist daher eher, dass die universelle Ausweitung des internationalen Systems einhergehend mit medialer Globalisierung sowie die Verregelung und Verrechtlichung der politischen Praktiken überhaupt erst die Anknüpfungspunkte für eine systematische Herrschaftsperspektive hervorgebracht hat, d.h. den diskursiven Rahmen bereitstellt, in dem wir bestimmte Praktiken als Ausdruck von Herrschaft und legitimationsbedürftig beschreiben können.

Ein Indiz dafür könnte sein, dass auch der Widerstand gegen diese Form globaler Politik ansteigt, seien es die teils auch gewaltsamen Proteste im Umfeld von G7/8Treffen oder WTO-Verhandlungen, die mit dem Battle von Seattle in den 1990er Jahren ihren Anfang nahmen (vgl. Andretta et al. 2006), die religiös konnotierten Proteste um die Mohammed-Karikaturen, die offenen Konflikte um die Atomprogramme in Nordkorea und Iran (Müller 2008), das Nein der Franzosen und Niederländer zur Europäischen Integration (Mair 2007), die massiven Proteste angesichts des Irakkrieges, die anhaltende Kontroverse um den Internationalen Strafgerichtshof innerhalb der Afrikanischen Union oder die Zunahme des transnationalen Terrorismus (Munck 2007). Mit ihnen allen verbindet sich mehr oder weniger explizit eine Kritik an der gegenwärtigen Ordnung internationaler Institutionen und politischer Praktiken, wenn auch aus ganz unterschiedlichen Richtungen, sei es als Ausdruck einer Hegemonie des Westens, des Kapitalismus, der technokratischen Ferne oder 
demokratischer Entmündigung. ${ }^{12}$ Vollkommen unabhängig davon, ob sich die Sphäre globaler Politik also tatsächlich dramatisch gewandelt hat, gilt, dass so lange sie zunehmend als Herrschaftsordnung und als Form der Beherrschung wahrgenommen wird, sich daraus wirkmächtige politische Konsequenzen und grundlegende Fragestellungen ergeben, die eine Internationale Politische Theorie aufzugreifen aufgefordert ist.

\section{Internationale Politische Theorie als Antwort auf Herrschaft}

Wenn meine Vermutung halbwegs zutreffend ist, dann sind die drängendsten Fragen, denen sich eine Internationale Politische Theorie stellen muss, die nach dem Charakter und der Legitimation bzw. Legitimationsfähigkeit der globalen Herrschaftsordnung. ${ }^{13}$ Da Herrschaft ein in den Internationalen Beziehungen lange Zeit kaum genutztes Konzept war, fehlt uns schon das Wissen um den Charakter der Herrschaftsordnung. ${ }^{14}$ Die spärliche Forschung dazu beschreibt sie entweder als hegemoniale Ordnung oder Empire, etwa im Rahmen poststrukturalistischer und postmarxistisch inspirierter Analysen (Hardt/Negri 2001; Cox 1981) oder-sogleich normativ gewendet - als heterarchische Ordnung, die im Modus der diskursiven Verständigung operiert (etwa Neyer 2004). In welchen Formen sich Herrschaft daher materialisiert, mit welchen Mechanismen sie operiert, welche Effekte sie wo und auf welche Akteure zeitigt und wie sich die Unterschiede in der Herrschaftsausübung in unterschiedlichen Sektoren und Konstellationen erklären lassen, bedarf einer empirisch-phänomenologischen und normativ-kritischen Betrachtungsweise. Diese kommt kaum ohne die begriffliche Reflexion seitens der Politischen Theorie aus, erfordert aber insbesondere gesellschaftstheoretische Analysen, die sich gegen den Nihilismus manch poststrukturalistischer und postmarxistischer Analysen behaupten können (ähnlich Brunkhorst 2010). ${ }^{15}$

Umgekehrt scheint die Legitimation und Legitimationsfähigkeit der globalen Herrschaftsordnung bereits gut erforscht zu sein. Spätestens seit den 1990er Jahren wird die Debatte sowohl in den Internationalen Beziehungen wie auch der Politischen Theorie geführt, ob eine demokratische Legitimation globalen Regierens überhaupt möglich (Dahl 1999; Scharpf 2004; Neyer 2010), nötig (Moravcsik 2004; 2006) und wenn, in welcher institutionellen Ausgestaltung sie am plausibelsten zu konzipieren sei (Wolf 2000; Abromeit 2002; Dingwerth 2007; Nanz/Steffek 2005; Buchanan/

12 Vgl. etwa die Arbeiten zu Politisierung und Resistance von Zürn et al. (2007); Nonhoff et al. (2009); Maiguashca (2003); Andretta et al. (2006); Munck (2007).

13 Wenngleich zugespitzt auf die normative Erörterung, beschreibt Rainer Forst die Erforschung der transnationalen Herrschaft als eine der zentralen Aufgaben der Internationalen Politischen Theorie (Forst 2010).

14 Wobei hier einschränkend zu bemerken ist, dass dies nicht in gleicher Weise für die Europäische Union gilt, deren sui generis Charakter als normative politische Ordnung früh und extensiv debattiert wurde.

15 Der Versuch, systematisch eine solche Perspektive auf Herrschaft und Kritik zu entwickeln, findet momentan in Frankfurt in mehreren Forschungsgruppen statt. 
Keohane 2006). Insbesondere die deliberative Demokratietheorie, die sich - zumindest auf den ersten Blick - aus der Verklammerung mit dem Nationalstaat und seinem spezifischen Institutionengefüge leicht lösen kann, hat diese Debatte enorm vorangebracht und bereits enge Bezüge zwischen Politischer Theorie und Internationalen Beziehungen hervorgebracht (Göhler et al. 2009; vgl. Niesen/Herborth 2007).

Die Frage nach der Legitimation internationaler Herrschaft ist aber immer noch zu beschränkt: Sowohl hinsichtlich der Frage, ob es jenseits oder im Verbund mit Demokratie alternative Legitimationskonzepte gibt (vgl. aber Neyer 2010) als auch bezüglich der generellen Anwendbarkeit von Demokratiemodellen, was wiederum auf die notwenige Forschung zum Charakter der Herrschaftsordnung verweist.

Wenn es richtig ist, dass sich mehr Widerstand und Widerspruch gegen internationalen Institutionen und Normen regt, muss als Frage in den Blickpunkt rücken, wie die globale Herrschaftsordnung von Seiten der Betroffenen wahrgenommen wird. Welche Art von Kritik wird woran formuliert, welche politischen Alternativen werden thematisiert? Aus einer Ordnungsperspektive ist empirisch zu fragen, wie diese Ordnung auf Dissens reagiert und normativ zu reflektieren, wie sie reagieren sollte. Wie, wo und durch wen wird Dissens geäußert und in die Entscheidungszentren gebracht, welche Charakteristika entscheiden darüber, ob Dissens ernst genommen wird, d.h. in die Entscheidungsfindung einbezogen wird oder nicht? Hat die Herrschaftsordnung überhaupt Räume und Kanäle für Dissens oder genauer: Opposition? Damit einher geht schließlich die Frage, inwiefern die gängigen governance- und demokratietheoretischen Modelle, die in der Debatte flottieren, überhaupt auf diese Fragen reagieren können (vgl. dazu Wiener 2010). Gilt für (normative) Global Governance-Konzepte, dass sie tendenziell konsensbasierte Politikmodelle prämieren, ${ }^{16}$ trifft gleiches in abgeschwächter Form auch für die gängigen Demokratiemodelle zu. Auch sie sind darauf ausgerichtet, Verfahren zu begründen, die Konsens hervorbringen oder zumindest befördern können (vgl. die zugespitzte Kritik bei Mouffe 2005). Ähnlich wie für Souveränität (Krasner 1999) dürfte auch für das klassische Prinzip des Staatenkonsenses als Entscheidungsgrundlage internationaler Politik gelten, dass es bestenfalls als organized hypocrisy zu verstehen ist. Wenn diese organisierte Heuchelei immer offener aufbricht und Dissens sich zunehmend radikalisiert, dann lautet die Aufgabe ernsthaft zu prüfen, ob die Suche nach konsensbasierten Verfahren und Institutionen nicht generell in die falsche Richtung geht und wir unsere Modelle nicht eher darauf ausrichten müssen, wie Dissens produktiv ausgehalten und ausgestaltet werden kann (vgl. O’Neill et al. 2008; auch Forst 2010 und Wiener 2010)? Wie lässt sich mit anderen Worten ein institutioneller Ort für Opposition denken in einem System, das kein Entscheidungszentrum kennt? Wie lässt sich verhindern, dass Opposition sich radikalisiert oder gar in Gewalt umschlägt? Jenseits der klassisch liberalen Modelle muss über alternative Theorieansätze nachgedacht werden und auch hier können sich Politische Theorie und Internationale Beziehungen vielfältige Anregungen geben.

$16 \mathrm{Zu}$ Recht weist daher Brunkhorst in seinem Beitrag darauf hin, dass für viele der gegenwärtigen normativen Modelle die »Widerständigkeit struktureller Herrschafts- und Klassenverhältnisse dann bestenfalls als restriktive Randbedingung politischen Handelns [erscheint]《 (Brunkhorst 2010: 299). 
Moderne, republikanische demokratietheoretische Modelle setzen Herrschaft und Beherrschung in das Zentrum ihrer Modelle (Pettit 2010; Rosanvallon 2008), werden aber bislang kaum in den Internationen Beziehungen rezipiert. Poststrukturalistische Theorien bieten mit ihrem Fokus auf Konflikt als bestimmendes Merkmal des Politischen in Form der aversiven oder agonalen Demokratietheorien (vgl. etwa Mouffe 2005; Norval 2007; im Überblick Marchart 2010) zumindest mögliche Fluchtwege aus der Konsensverengung, die Global Governance auszeichnet. Gerade die Debatte innerhalb der Politischen Philosophie und Politischen Theorie um die Konkurrenz oder Komplementarität zwischen deliberativen und agonalen Demokratietheorien ist hierzu instruktiv, aber wiederum kaum rezipiert (vgl. etwa Urbinati 2010; Knopf 2007; Honig 2007). Damit verbindet sich keineswegs eine Zurückweisung liberaler Demokratietheorien oder breiter, liberaler Weltordnungsentwürfe, die unverkennbar nach wie vor die Debatte über globale Politik dominieren (vgl. Geis et al. 2006), sondern nur die kritische Reflexion von deren Implikationen und Reichweite. Der nahezu ungebrochene Optimismus liberaler Weltordnungsentwürfe, wie er sich besonders eindrücklich in der Kosmopolitismus-Debatte einerseits (stellvertretend etwa Archibugi 1998) oder der Global Governance-Debatte andererseits zeigt (zuvorderst Slaughter 1995; 2004), aber auch in die Politik eingesickert ist, wird andernorts längst kritisch hinterfragt. Die anwachsende Forschung zu Liberalismus, Demokratie und Gewalt hat hinlänglich herausgearbeitet, dass sich im Kern dieser liberalen Ordnung ein erhebliches Sprengpotenzial für eine friedliche Weltordnung verbirgt (Jahn 2005; Müller 2007), da liberale Demokratien - in wenngleich variierendem Ausmaß - dazu neigen, liberale Werte, Normen und Praktiken a priori als universell gültig zu unterstellen und daher zumindest eine Tendenz aufweisen, diese nötigenfalls auch gewaltsam durchzusetzen (Müller 2008; 2009; Geis et al. 2006). Darum ist auch eine Internationale Ethik gefragt, die den divergierenden Weltentwürfen und Wertvorstellungen nachspürt und nach alternativen Ordnungsmodellen sucht.

\section{Schluss}

An diesen nur kurz und eher unsystematisch andiskutierten Fragen wird bereits zweierlei deutlich: Zum einen sind sie kaum zu bearbeiten, ohne dass Internationale Beziehungen und Politische Theorie zusammen arbeiten, zum anderen sollte Internationale Politische Theorie als offenes Feld verstanden werden, das sich weder auf eine Internationale Ethik noch auf Demokratietheorie oder Gesellschaftstheorie beschränken lässt, sondern Bedarf für ganz unterschiedliche Zugriffe entwickelt.

Die aufgeworfenen Fragen stellen beide Teildisziplinen vor immense Herausforderungen. Für die Politische Theorie bedeuten sie, die eigenen Begriffe und normativen Konzepte aus ihrer Verklammerung mit dem Nationalstaat zu lösen und sich zugleich die Besonderheiten der politischen Praktiken jenseits des Staates - über IB- 
realistisch konnotierte Machtpolitik hinaus - zu erschließen. ${ }^{17}$ Für die Internationalen Beziehungen folgt aus ihnen, die hergebrachten Kategorien gründlich zu hinterfragen und sich auch jenseits einzelner Begriffsimporte ernsthaft auf die Diskussionen der Politischen Theorie einzulassen (vgl. auch Deitelhoff/Wolf 2009). Eine Eingrenzung der Internationalen Politischen Theorie auf bestimmte Formen theoretischer oder methodischer Zugänge ist daher kaum wünschenswert. Internationale Politische Theorie sollte sich vorrangig aus ihrem Gegenstand heraus definieren. Hegemoniale Grenzziehungen von jedweder Seite, die Internationale Politische Theorie als Feld der Politischen oder Moralphilosophie globaler Politik verstehen und gesellschaftstheoretische Fragen eher ausschließen wollen, ${ }^{18}$ oder aus den Internationalen Beziehungen, die eine Internationale Politische Theorie von vornherein als irrelevant abtun möchten, sind gleichermaßen problematisch. Während den einen über die Ausblendung empirischer Reflexion droht, ihren Gegenstand aus dem Auge zu verlieren, verlieren die anderen die Konzepte, mit denen sie ihren Gegenstand sinnhaft befragen könnten.

Internationale Politische Theorie fordert daher die Verschränkung, wenn nicht Verschmelzung der Erkenntnishorizonte, die aus den unterschiedlichen Sozialisationen und Fachtraditionen eine neue Verbindung schafft, ohne dass die eine die andere dominiert. Daraus folgt aber nicht, dass eine neue Sektion oder gar Teildisziplin zu etablieren ist. Die geforderte Verschmelzung betrifft zum einen nur einen kleinen Teil in beiden Teildisziplinen, nämlich diejenigen, die sich mit der globalen Ordnung, sei es als Herrschafts- oder Machtordnung, beschäftigen. Zum anderen beschränkt sie sich auf die Erkenntnishorizonte, lässt aber Platz für, ja benötigt gerade plurale und konkurrierende methodisch-theoretische Zugriffe.

Ob es gelingt, aus den bislang parallelen Universen ein gemeinsames zu schaffen, ist selbst mit diesen Einschränkungen offen. Die Zeichen dafür stehen aber nicht zu schlecht. Gerade die Demokratiedebatte hat die Auseinandersetzung zwischen Politischer Theorie und Internationalen Beziehungen weit vorangebracht; und auch der Widerhall auf die Frankfurter Tagung mit insgesamt 160 Anmeldungen ist - wie die Einrichtung einer eigenen Professur für Internationale Politische Theorie in Frankfurt - ein Signal dafür, dass die Verschmelzung vorangeht und die (teil-)disziplinären Grenzen durchlässiger werden.

\section{Literatur}

Abromeit, Heidrun 2002: Wozu braucht man Demokratie? Die postnationale Herausforderung der Demokratietheorie, Opladen.

17 Dass auch die Politische Theorie gefordert ist, sich mehr als bisher für die Erkenntnisse und Diskurse in den IB zu öffnen, wird auch an Brunkhorsts Beitrag deutlich, der zwar eine erheblich differenzierte Betrachtung der internationalen Beziehungen vorlegt, aber meint, dass die Internationalen Beziehungen nach wie vor vom Realismus dominiert seien (Brunkhorst 2010), was zumindest für den deutschsprachigen Raum kaum zu halten ist. Vgl. dagegen aber etwa den Beitrag von Niesen (2010).

18 Vgl. ECPR (2010) oder auch International Political Theory (2010). 
Albert, Mathias/Stichweh, Rudolf 2007 (Hrsg.): Weltstaat und Weltstaatlichkeit. Beobachtungen globaler politischer Strukturbildung, Wiesbaden.

Andretta, Massimiliano/della Porta, Donatella/Mosca, Lorenzo/Reiter, Herbert 2006: Globalization from Below: Transnational Activists and Protest Networks, Minneapolis, MN.

Archibugi, Daniele 1998: Principles of Cosmopolitan Democracy, in: Archibugi, Daniele/Held, David/Köhler, Martin (Hrsg.): Reimagining Political Community: Studies in Cosmopolitan Democracy, Stanford, CA, 198-228.

Arendt, Hannah 1970: Macht und Gewalt, München.

Beck, Ulrich 2007: Weltrisikogesellschaft, Frankfurt a. M.

Beitz, Charles 1979: Political Theory and International Relations, Princeton, NJ.

Brock, Lothar 2007: Universalismus, politische Heterogenität und ungleiche Entwicklung. Internationale Kontexte der Gewaltanwendung von Demokratien gegenüber Nichtdemokratien, in: Geis, Anna/Müller, Harald/Wagner, Wolfgang (Hrsg.): Schattenseiten des Demokratischen Friedens. Zur Kritik einer Theorie liberaler Außen- und Sicherheitspolitik, Frankfurt a. M., 45-68.

Brodocz, André/Schaal, Gary S. 2002: Einleitung, in: Brodocz, André/Schaal, Gary S. (Hrsg.): Politische Theorien der Gegenwart I, Opladen, 9-22.

Brown, Chris 1992: International Relations Theory: New Normative Approaches, New York, NY.

Brunkhorst, Hauke 2009 (Hrsg.): Demokratie in der Weltgesellschaft (Sonderband 18: Soziale Welt), Baden-Baden.

Brunkhorst, Hauke 2010: Neustart - Kritische Theorie Internationaler Beziehungen, in: Zeitschrift für Internationale Beziehungen 17: 2, 293-315.

Buchanan, Alan/Keohane, Robert O. 2006: The Legitimacy of Global Governance Institutions, in: Ethics and International Affairs 20: 4, 405-437.

Bull, Hedley 1977: The Anarchical Society: A Study of Order in World Politics, New York, NY.

Cox, Robert W. 1981: Social Forces, States and World Orders: Beyond International Relations Theory, in: Millennium 10: 2, 126-155.

Daase, Christopher 2003: Die Englische Schule, in: Schieder, Siegfried/Spindler, Manuela (Hrsg.): Theorien der Internationalen Beziehungen, Opladen, 227-252.

Dahl, Robert 1999: Can International Organizations be Democratic? A Sceptic's View, in: Shapiro, Ian/Hacker-Cordón, Casiano (Hrsg.): Democracy's Edges, Cambridge, MA, 19-36.

Deitelhoff, Nicole/Gosepath, Stefan/Niesen, Peter/Herborth, Benjamin 2010: Call for Papers zur Tagung »Internationale Politische Theorie«, 10.-12. Juni 2010 in Frankfurt a. M., in http:// www.unibw.de/ib-sektion/docs/cfp-int.pdf, 05.11.2010.

Deitelhoff, Nicole/Steffek, Jens 2009: Einleitung: Staatlichkeit ohne Staat?, in: Deitelhoff, Nicole/Steffek, Jens (Hrsg.): Was bleibt vom Staat? Demokratie, Recht und Verfassung im globalen Zeitalter, Frankfurt a. M., 7-34.

Deitelhoff, Nicole/Wolf, Klaus Dieter 2009: Der Widerspenstigen Selbst-Zähmung? Zur Professionalisierung der Internationalen Beziehungen in Deutschland, in: Politische Vierteljahresschrift 50: 3 (Sonderheft: Politikwissenschaft in Deutschland. Eine Bestandsaufnahme zu 50 Jahren PVS), 451-475.

Deutsch, Karl W. 1969: Politische Kybernetik. Modelle und Perspektiven, Rombach.

Dingwerth, Klaus 2007: The New Transnationalism: Transnational Governance and Democratic Legitimacy, Basingstoke.

Durchhardt, Heinz 1997: Balance of Power und Pentarchie 1700-1785 (Handbuch der Geschichte der internationalen Beziehungen, Bd. 4), Paderborn.

ECPR 2010: Standing Group on International Political Theory, in: http://www.essex.ac.uk/ecpr/ standinggroups/ipt/ipt.aspx; 25.9.2010.

Forst, Rainer 2010: Was ist und was soll Internationale Politische Theorie?, in: Zeitschrift für Internationale Beziehungen 17: 2, 355-363.

Frost, Mervyn 1986: Towards a Normative Theory of International Relations, Cambridge. 
Gadamer, Hans-Georg 1990: Wahrheit und Methode. Grundzüge einer philosophischen Hermeneutik, Tübingen.

Gadamer, Hans-Georg 1993: Wahrheit und Methode. Ergänzung und Register, Tübingen.

Geis, Anna/Brock, Lothar/Müller, Harald 2006 (Hrsg.): Looking at the Dark Side of Democratic Peace, Basingstoke.

Gill, Stephen 2003: Power and Resistance in the New World Order, Basingstoke.

Göhler, Gerhard/Iser, Matthias/Kerner, Ina 2009: Entwicklungslinien der Politischen Theorie in Deutschland seit 1945, in: Politische Vierteljahresschrift 50: 3 (Sonderheft: Politikwissenschaft in Deutschland. Eine Bestandsaufnahme zu 50 Jahren PVS), 372-407.

Hardt, Michael/Negri, Antonio 2001: Empire, Cambridge, MA.

Hasenclever, Andreas 2002: Die Macht der Moral in der Internationalen Politik, Frankfurt a. M.

Held, David 1995: Democracy and the Global Order: From the Modern State to Cosmopolitan Governance, Cambridge.

Held, David/McGrew, Anthony 2002: Introduction, in: Held, David/Mc Grew, Anthony (Hrsg.): Governing Globalization: Power, Authority, and Global Governance, Cambridge, 1-25.

Hettne, Björn/Söderbaum, Fredrik 2008: The Future of Regionalism: Old Divides, New Frontiers, in: Cooper, Andrew F./Hughes, Christopher W./Lombaerde, de Phillippe (Hrsg.): Regionalisation and Global Governance: The Taming of Globalisation?, Abingdon, 61-79.

Higgott, Richard 2000: Contested Globalization: the Changing Context and Normative Challenges, in: Review of International Studies 26: 5, 131-153.

Hirst, Paul/Thompson, Grahame 1996: Globalization in Question, Cambridge.

Honig, Bonnie 2007: Between Decision and Deliberation: Political Paradox in Democratic Theory, in: American Political Science Review 101: 1, 1-17.

Hurrell, Andrew 2007: On Global Order: Power, Values and the Constitution of International Society, Oxford.

Hurrelmann, Achim/Schneider, Steffen/Steffek, Jens 2007 (Hrsg.): Legitimacy in an Age of Global Politics, Houndmills.

International Political Theory 2010: Webseite, in: http://international-political-theory.net/; 25.9.2010.

Jahn, Beate 2005: Kant, Mill, and Illiberal Legacies in International Affairs, in: International Organization 59: 4, 177-207.

Kahler, Miles 1995: International Institutions and the Political Economy of Integration, Washington, D.C.

Kapstein, Ethan 2000: Winners and Losers in the Global Economy, in: International Organization 54: 2, 359-384.

Kessler, Oliver 2010: Internationale Politische Theorie: Fehlentwicklung oder fehlende Entwicklung der IB?, in: Zeitschrift für Internationale Beziehungen 17: 2, 317-334.

Knopf, Andrew 2007: Debate: Agonism as Deliberation: On Mouffe's Theory of Democracy, in: The Journal of Political Philosophy 15: 1, 115-126.

Kohler-Koch, Beate/Rittberger, Berthold 2007 (Hrsg.): Debating the Democratic Legitimacy of the European Union, Lanham, MD.

Krasner, Stephen 1999: Sovereigtny: Organized Hypocrisy, Princeton, NJ.

Linklater, Andrew 1998: The Transformation of Political Community, Cambridge.

Luhmann, Niklas 2003: Macht, Stuttgart.

Maiguashca, Bice 2003: Governance and Resistance in World Politics, in: Review of International Studies 29: 1, 3-28.

Mair, Peter 2007: Political Opposition and the European Union, in: Government and Opposition 42: 1, 1-17.

Marchart, Oliver 2010: Die politische Differenz, Frankfurt a. M.

Mayer, Peter 2006: Macht, Gerechtigkeit und internationale Kooperation. Eine regimeanalytische Studie zur internationalen Rohstoffpolitik, Baden-Baden. 
Mayntz, Renate 2008: Von der Steuerungstheorie zu Global Governance, in: Schuppert, Gunnar Folke/Zürn, Michael (Hrsg.): Governance in einer sich wandelnden Welt, Wiesbaden, 43-60.

Moravcsik, Andrew 2004: Is there a »Democratic Deficit« in World Politics? A Framework for Analysis, in: Government and Opposition 39: 2, 336-363.

Moravcsik, Andrew 2006: What Can We Learn from the Collapse of the European Constitutional Project?, in: Politische Vierteljahresschrift 47: 2, 219-241.

Mouffe, Chantal 2005: On the Political, London.

Munck, Ronaldo 2007: Globalization and Contestation: The New Great Counter-Movement, Abingdon.

Müller, Harald 1994: Internationale Beziehungen als kommunikatives Handeln, in: Zeitschrift für Internationale Beziehungen 1: 1, 15-44.

Müller, Harald 2004: Think Big! Der 11. September und seine Konsequenzen für die Internationalen Beziehungen, in: Zeitschrift für Internationale Beziehungen 11: 1, 123-134.

Müller, Harald 2007: Vorüberlegungen zu einer Theorie der Ambivalenz liberal-demokratischer Außen- und Sicherheitspolitik, in: Geis, Anna/Müller, Harald/Wagner, Wolfgang (Hrsg.): Schattenseiten des Demokratischen Friedens, Frankfurt a. M., 287-312.

Müller, Harald 2008: Wie kann eine neue Weltordnung aussehen? Wege in eine nachhaltige Politik, Frankfurt a. M.

Müller, Harald 2009: Staatlichkeit ohne Staat - ein Irrtum aus der europäischen Provinz? Limitierende Bedingungen von Global Governance in einer fragmentierten Welt, in: Deitelhoff, Nicole/Steffek, Jens (Hrsg.): Was bleibt vom Staat? Demokratie, Recht und Verfassung im globalen Zeitalter, Frankfurt a. M., 221-258.

Münkler, Herfried 2002: Die neuen Kriege, Hamburg.

Nanz, Patricia/Steffek, Jens 2005: Assessing the Democratic Quality of Deliberation in International Governance: Criteria and Research Strategies, in: Acta Politica 40: 3, 368-383.

Neyer, Jürgen 2002: Politische Herrschaft in nicht-hierarchischen Mehrebenensystemen, in: Zeitschrift für Internationale Beziehungen 9: 1, 9-38.

Neyer, Jürgen 2004: Postnationale Politische Herrschaft. Vergesellschaftung und Verrechtlichung jenseits des Staates, Baden-Baden.

Neyer, Jürgen 2010: Justice, Not Democracy: Legitimacy in the European Union, in: Journal of Common Market Studies, i.E.

Niesen, Peter 2007: Politische Theorie als Demokratiewissenschaft, in: Buchstein, Hubertus/ Göhler, Gerhard (Hrsg.): Politische Theorie und Politikwissenschaft, Wiesbaden, 126-155.

Niesen, Peter 2010: Internationale Politische Theorie: Eine disziplinengeschichtliche Einordnung, in: Zeitschrift für Internationale Beziehungen 17: 2, 267-277.

Niesen, Peter/Herborth, Benjamin 2007 (Hrsg.): Anarchie der kommunikativen Freiheit, Frankfurt a. M.

Nonhoff, Martin/Gronau, Jennifer/Nullmeier, Frank/Schneider, Steffen 2009: Zur Politisierung internationaler Institutionen. Der Fall G8, in: Zeitschrift für Internationale Beziehungen 16: 2, 233-263.

Norval, Aletta 2007: Aversive Democracy, Cambridge.

O'Neill, Daniel I./Shanley, Mary Lyndon/Young, Iris Marion 2008 (Hrsg.): Illusion of Consent: Engaging with Carole Pateman, University Park, PA.

Osterhammel, Jürgen/Petersson, Niels P. 2004: Geschichte der Globalisierung, München.

Papadopoulos, Yannis 2004: Governance und Demokratie, in: Benz, Arthur (Hrsg.): Governance. Regieren in komplexen Regelsystemen, Wiesbaden, 215-238.

Pettit, Philip 2010: A Republican Law of Peoples, in: European Journal of Political Theory 9: 1, 70-94.

Rittberger, Volker 1990: Editorisches Vorwort, in: Rittberger, Volker (Hrsg.): Theorien der Internationalen Beziehungen (Sonderheft 21/1990, Politische Vierteljahresschrift), Opladen, 7-16.

Rosanvallon, Pierre 2008: Counter-Democracy: Politics in an Age of Distrust, Cambridge. 
Ruggie, John Gerard 1983: International Regimes, Transaction and Change: Embedded Liberalism in the Postwar Economic Order, in: Krasner, Stephen D. (Hrsg.): International Regimes, Ithaca, NY, 195-231.

Scharpf, Fritz W. 2004: Legitimationskonzepte jenseits des Nationalstaats, MPIfG Working Paper $04 / 6$.

Schmalz-Bruns, Rainer 1995: Die Theorie Kommunikativen Handelns - eine Flaschenpost, in: Zeitschrift für Internationale Beziehungen 2: 1, 347-361.

Slaughter, Anne-Marie 1995: International Law in a World of Liberal States, in: European Journal of International Law 6: 4, 503-538.

Slaughter, Anne-Marie 2004: A New World Order, Princeton, NJ.

Steffek, Jens 2009: Die Legitimation internationalen Regierens: Vom Staatenkonsens zum Bürgerkonsens, in: Deitelhoff, Nicole/Steffek, Jens (Hrsg.): Was bleibt vom Staat? Demokratie, Recht und Verfassung im globalen Zeitalter, Frankfurt a. M., 187-220.

Urbinati, Nadia 2010: Unpolitical Democracy, in: Political Theory 38: 1, 65-92.

Weber, Max 1972: Wirtschaft und Gesellschaft, Tübingen.

Wiener, Antje 2010: Zur normativen Wende in den IB: Triangulation of a Different Kind, in: Zeitschrift für Internationale Beziehungen 17: 2, 335-354.

Wolf, Klaus Dieter 2000: Die neue Staatsräson. Zwischenstaatliche Kooperation als Demokratieproblem in der Weltgesellschaft, Baden-Baden.

Zangl, Bernhard/Zürn, Michael 2003: Frieden und Krieg. Sicherheit in der nationalen und postnationalen Konstellation, Frankfurt a. M.

Zürn, Michael 1998: Regieren jenseits des Nationalstaats, Frankfurt a. M.

Zürn, Michael/Binder, Martin/Ecker-Ehrhardt, Matthias/Radtke, Katrin 2007: Politische Ordnungsbildung wider Willen, in: Zeitschrift für Internationale Beziehungen 14: 1, 129-164. 